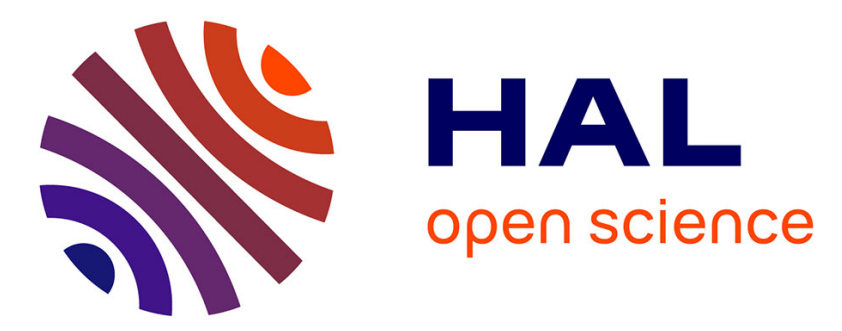

\title{
Epitaxial growth of honeycomb-like stanene on $\mathrm{Au}(111)$ Wenhui Pang, Kazuki Nishino, Tsuyoshi Ogikubo, Masaaki Araidai, Masashi Nakatake, Guy Le Lay, Junji Yuhara
}

\section{To cite this version:}

Wenhui Pang, Kazuki Nishino, Tsuyoshi Ogikubo, Masaaki Araidai, Masashi Nakatake, et al.. Epitaxial growth of honeycomb-like stanene on $\mathrm{Au}(111)$. Applied Surface Science, 2020, 517, pp.146224. 10.1016/j.apsusc.2020.146224 . hal-03202614

\section{HAL Id: hal-03202614 https://hal.science/hal-03202614}

Submitted on 20 Apr 2021

HAL is a multi-disciplinary open access archive for the deposit and dissemination of scientific research documents, whether they are published or not. The documents may come from teaching and research institutions in France or abroad, or from public or private research centers.
L'archive ouverte pluridisciplinaire HAL, est destinée au dépôt et à la diffusion de documents scientifiques de niveau recherche, publiés ou non, émanant des établissements d'enseignement et de recherche français ou étrangers, des laboratoires publics ou privés. 


\title{
Epitaxial growth of honeycomb-like stanene on Au(111)
}

\author{
Wenhui Pang $^{\mathrm{a}}$, Kazuki Nishino ${ }^{\mathrm{a}}$, Tsuyoshi Ogikubo ${ }^{\mathrm{a}}$, Masaaki Araidai ${ }^{\mathrm{a}, \mathrm{b}, \mathrm{c}}$, Masashi \\ Nakatake $^{d}$, Guy Le Lay ${ }^{\mathrm{e}}$, Junji Yuhara ${ }^{\mathrm{a}, *}$ \\ a Department of Energy Engineering, Nagoya University, Nagoya 464-8603, Japan \\ b Institute for Advanced Research, Nagoya University, Nagoya 464-8601, Japan \\ C Institute of Materials and Systems for Sustainability, Nagoya University, Nagoya 464-8601, \\ Japan \\ d Aichi Synchrotron Radiation Center, Aichi Science \& Technology Foundation, Seto, Aichi \\ 489-0965, Japan
}

e Aix-Marseille Université, CNRS, PIIM UMR 7345, 13397 Marseille Cedex, France

\begin{abstract}
Stanene, which is predicted to be a quantum spin Hall topological insulator with tunable topological state, seems to be the most promising candidate of the post-graphene elemental two-dimensional (2D) materials. Here, we prepared epitaxial honeycomb-like stanene on gold (111) substrates and investigated its superstructure by Low Energy Electron Diffraction and Scanning Tunneling Microscopy. Angle-Resolved PhotoEmission Spectroscopy was applied to explore the electronic structures, further confirmed by first principles calculations. The stanene- like sheet forms a nearly planar structure on the $\mathrm{Au}(111)$ surface with a " $2 \times \sqrt{3}$ " superstructure in large surface areas. Core-level spectroscopy reveals that the stanene-like sheet lays almost directly on the Au(111) surface. This is consistent with DFT calculations of the atomic structure. A characteristic 2D band with parabolic dis- persion is observed.
\end{abstract}

\section{Introduction}

The success of graphene has driven extensive attention to other two- dimensional (2D) materials, due to outstanding properties, such as to- pological insulating character [1], Quantum Spin Hall (QSH) effect [2,3], and enhanced thermoelectricity [4]. Unfortunately, however, most available materials with such properties are until now often based on multicomponent materials with different sublattices or complex alloys. These are still challenging to fabricate in terms of large-scale production. Among various post-graphene elemental 2D materials [5], one of them, stanene, the tin-based analog, attracted special attention due to its Dirac cone-like energy dispersion and large spin-orbit strength, permitting to envision exciting emerging properties. Typi- cally, it has been predicted that stanene with a large gap 2D QSH state can enable dissipationless electric conduction at room temperature (RT) [6-8]. Several theoretical and experimental efforts have been carried out on stanene [9-11], e.g., stanene with a $0.44 \mathrm{eV}$ band gap, has been grown on $\operatorname{lnSb}(111)$ recently [12].

The first realization of a highly buckled stanene-like overlayer has been achieved on a $\mathrm{Bi} 2 \mathrm{Te} 3$ topological insulator substrate [13]. Then further researches have been devoted to exploring the distinct elec- tronic properties of stanene formed on different substrates, such as semiconducting $\operatorname{InSb}(111), \operatorname{SiC}(0001)$ and MoS2 [11,14,15], semimetals $\mathrm{Sb}(111)$ [16], and metallic $\mathrm{Ag}(111)$ [17], $\mathrm{Cu}(111)$ [18]; for interesting reviews, see Refs. [19] and [20]. Searching for alternative substrate materials, recent theoretical works especially predicted interesting 
possibilities with the $\mathrm{Au}(111)$ surface [21], while density functional theory (DFT) calculations indicate that low-buckled stanene is more stable than highly buckled stanene [3].

A few groups have studied the growth of tin on gold (111) surfaces by different methods [2227]. However, the experimental realization of stanene remains challenging. To the best of our knowledge planar honeycomb-like tin with a " $2 \times \sqrt{3}$ " superstructure on the $\mathrm{Au}(111)$ surface, as will be shown here, has never been reported; hence, we will describe its realization and properties in this paper.

This novel tin overlayer features a low-buckled lattice with even- tually three $\mathrm{Sn}$ atoms per unit cell arranged in a honeycomb-like structure, as derived from scanning tunneling microscopy (STM), low- energy electron diffraction (LEED) and synchrotron radiation core-level spectroscopy (CLS), along with DFT calculations of the atomic geo- metry. The electronic properties were explored by angle-resolved photoemission spectroscopy (ARPES), and compared to DFT band structure calculations.

\section{Experiment and calculations}

We performed our experiments in two different ultra-high vacuum vessels. The first one at Nagoya University, is equipped with a LEED apparatus operating with a LaB6 filament and a UHV Omicron STM-1 system. The second one used for the CLS and ARPES experiments, is at the Aichi synchrotron radiation center. It hosts a MCP-LEED system and a hemispherical analyzer with a $200 \mathrm{~mm}$ radius (MB Scientific AB A-1) and wide-angle electron lens. Both vessels consist of a preparation chamber and an analysis chamber with base pressure lower than $10^{-10}$ mbar.

After sputtering the $\mathrm{Au}(111)$ surfaces by $700 \mathrm{eV} \mathrm{Ar}^{+}$ions at $\mathrm{RT}$, an annealing at around 600 ${ }^{\circ} \mathrm{C}$ was performed. Temperatures were mon- itored with a radiation thermometer and a type $\mathrm{K}$ thermocouple fixed on the base plate of the sample holder. After annealing, Auger electron spectroscopy (AES) was performed at RT to check for surface cleanli- ness. Then the LEED patterns showed sharp reconstruction spots of the well-known $\mathrm{Au}(111) 22 \times \sqrt{3}$ herringbone clean superstructure.

Tin was deposited onto the $A u(111)$ surfaces at $R T$ in either UHV vessel with a deposition rate of approximately 0.13 monolayer $(\mathrm{ML}) \mathrm{min}^{-1}$ (the rate was measured by using a quartz crystal monitor). The atomic density of a $\mathrm{Au}(111)$ plane is $1.391 \times 10^{15}$ atoms $\mathrm{cm}^{-2}$; we use this value to define $1 \mathrm{ML}$ Sn coverage. The deposition rate had been calibrated by Rutherford backscattering spectroscopy (RBS); the methodology has been published in Refs. [28] and [29]. The amount of deposited $\mathrm{Sn}$ is $0.6 \mathrm{ML}$; errors are less than $10 \%$ in mentioned coverage ratios.

In each vessel, the LEED and STM observations were performed at RT. Sharp LEED patterns were obtained with 46-70 eV primary beam energies. All STM images were acquired with W tips. The CL and ARPES spectra were recorded at about $10 \mathrm{~K}$, as the gold specimen was mounted on a liquid-helium-cooled cryostat with 4-axis pulse motor control.

The DFT calculations were performed by the QuantumATK [30], in which wave functions are expressed by the linear combination of pseudo atomic orbitals. The interactions between ionic core and va- lence electrons were described by ONCV-type pseudopotentials of the PseudoDojo project $[31,32]$ and PBE-type exchange-correlation energy functional was also employed [33]. The lateral size of the $\mathrm{Au}(111)$ si- mulation cell was $(a, b)=(5.768,4.995)$ in 
unit of $\AA$; periodic boundary conditions were imposed along both directions. In the c di- rection, one side of the $\mathrm{Au}(111)$ slab consisting of 14 atomic layers was coupled to the semi-infinite $\mathrm{Au}(111)$ electrode as the boundary condi- tion by the Green's function technique [34], and the opposite side had a vacuum region of $15 \AA$. The atomic configuration except for three layers close to the electrode was relaxed until the forces acting on atoms be- came smaller than 10 meV/A. The cutoff energy for space discretization was taken to be $2721.14 \mathrm{eV}$. The Brillouin zone of the unit cell was sampled with a $5 \times 6 \times 1 \Gamma$-center k-point grid.

\section{Results and discussion}

In order to study the growth of tin on the Au(111) surface, at first LEED and STM observations have been carried out. Fig. 1(a) and (b) show the LEED pattern and STM images of the well characterized $\mathrm{Au}(111) 22 \times \sqrt{3}$ herringbone reconstruction [35]. Terraces are separated by atomic steps $0.23 \mathrm{~nm}$ in height, as shown by the $A B$ profile in Fig. $1(b)$ and (f).
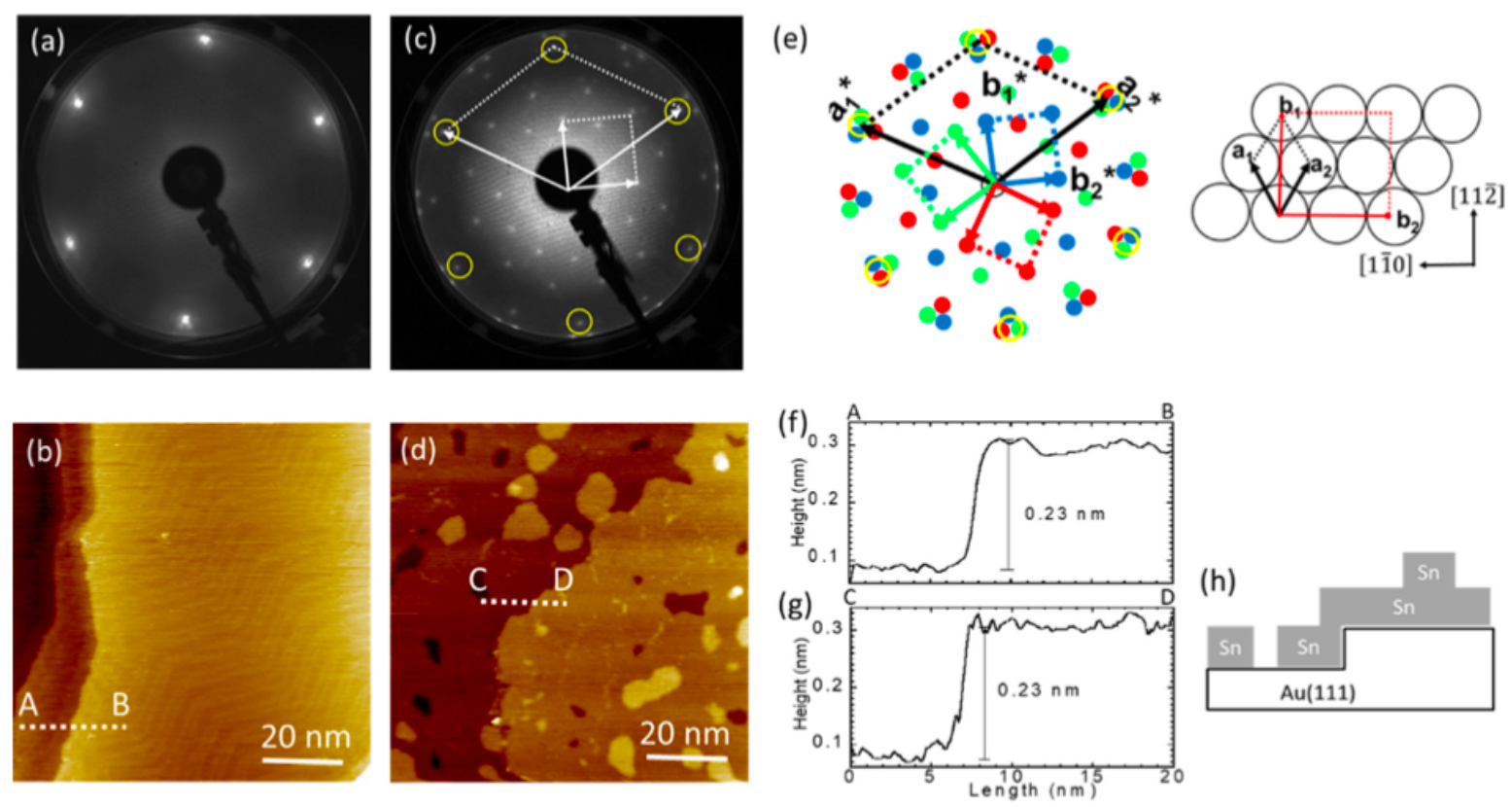

Fig. 1. (a) LEED pattern of the pristine $A u(111)$ surface $(55 \mathrm{eV})$. (b) large-scale STM image of the bare $\mathrm{Au}(111)$ surface showing the herringbone superstructure $(\mathrm{Us}=1.0 \mathrm{~V}, \mathrm{I}=0.2 \mathrm{~A}$ ). (c) and $(\mathrm{d})$ LEED pattern $(55 \mathrm{eV})$ and the large-scale STM image $(\mathrm{Us}=1.0 \mathrm{~V}, \mathrm{I}=0.2 \mathrm{nA})$ of 0.6 ML Sn deposited onto the Au(111) surface at RT. (e) Simulated LEED pattern of the " $2 \times \sqrt{3}$ " superstructure. (f) and (g) Sectional profiles along the A-B and C-D lines in (b) and (d). (h) Growth model of tin on the Au(111) surface.

The LEED pattern and the large scale STM image $(100 \mathrm{~nm} \times 100 \mathrm{~nm})$ of the $0.60 \mathrm{Sn} \mathrm{ML}$ on the $\mathrm{Au}(111)$ surface are displayed in the Fig. 1(c) and (d). After deposition, the LEED patterns exhibit the characteristic diffraction pattern of the " $2 \times \sqrt{3}$ " super- structure, which is simulated in Fig. 1(e) with three symmetry equivalent domains. Along the[11 0$]$ and[112 ] directions the new lattice constants b1 and b2 are expanded/reduced with respect to commen- surate 2a1 and $\sqrt{3} a_{2}$ values of the unreconstructed $A u(111) 1 \times 1$ sur- face, that is $|b 1|=1.1 \times \sqrt{3}|a 1|$ and $|\mathrm{b} 2 \mathrm{I}=0.95 \times 2 \mathrm{la2}|$, the super-

structure matrix being $\left[\begin{array}{ll}1.9 & 0.0 \\ 1.1 & 2.2\end{array}\right]$. 
This is why we name the superstructure as " $2 \times \sqrt{ } 3$ ", since it is in reality incommensurate. This may be related to the uniaxial compression of the gold surface atoms along the [11-0] direction [35].

In Fig. 1(d), the smooth terraces no longer show any signature of the herringbone reconstruction and no 3D islands are observed. Locally, bare Au(111) is recognized as black holes. These terraces are separated by steps with a uniform height of $0.23 \mathrm{~nm}$ as shown in Fig. 1(g), indicating a $\mathrm{Au}(111)$ step below the tin overlayer. Clearly the $\mathrm{Sn}$ atoms form an adlayer wetting the $\mathrm{Au}(111)$ surface; the overlayer film is uniform over hundred nanometers with just some second layer patches, as displayed in Fig. 1(h).

The high-resolution STM image displayed in Fig. 2(a) reveals the atomic arrangement of the " $2 \times \sqrt{3}$ " superstructure. It points to two different local arrangements, namely chain-like within the yellow rec- tangles and honeycomb-like (hexagonal motifs are aligned only in one direction) within the black squares, as illustrated in Fig. 2(b) and (e), respectively, with possibly three equivalent orientations. In the black square areas, the protrusions appear denser than in the yellow rectangle ones, where they look like zigzag chains.

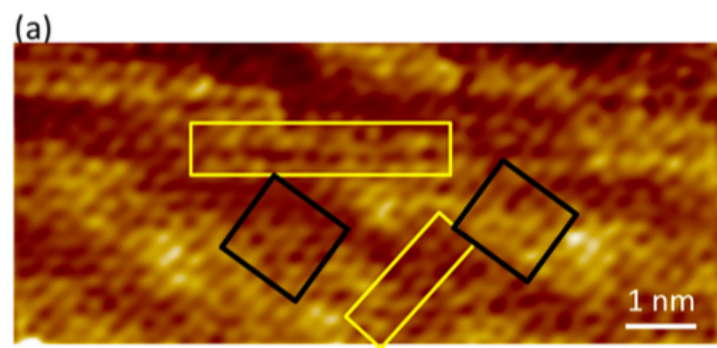

(b)

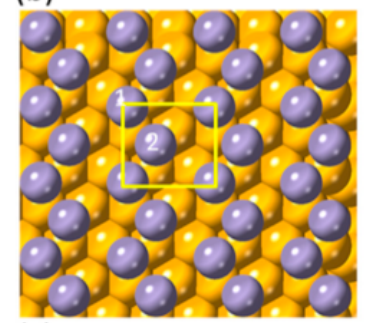

(c)

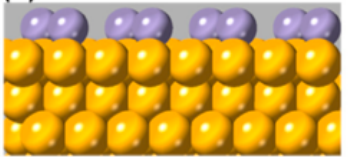

(d)

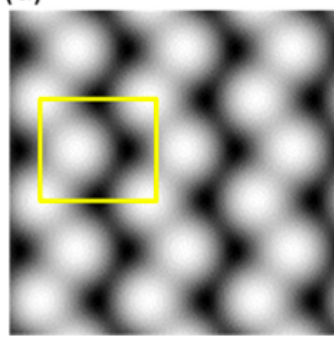

(e)

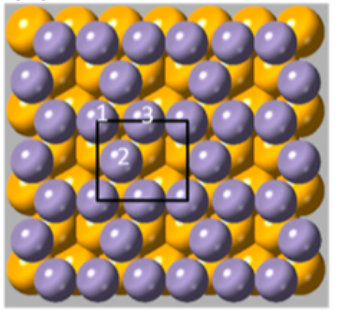

(f)

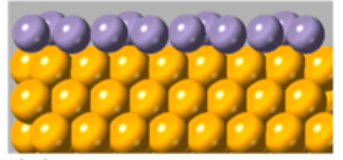

(g)

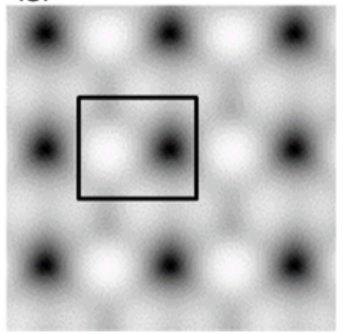

Fig. 2. (a) High-resolution STM image of the Sn covered $A u(111)$ surface $(0.6 \mathrm{ML}$; Us $=0.3 \mathrm{~V}$, $\mathrm{I}=0.2 \mathrm{nA})$. (b) and (c) Top and side views of the zigzag chain- like $(2 \times \sqrt{3})$ approximant structure. (e) and (f) Top and side views of the honeycomb-like $(2 \times \sqrt{3})$ approximant structure. Yellow and gray balls denote $\mathrm{Au}$ and $\mathrm{Sn}$ atoms, respectively. Yellow and black squares indicate the $(2 \times \sqrt{3})$ unit cell. $(\mathrm{d})$ and $(\mathrm{g})$ are the simulated STM images for the zigzag chain-like and honeycomb-like structures, respectively. 
Assuming commensurate $(2 \times \sqrt{3})$ cells, representing approximants, we propose two atomic models differing in tin coverage ratios, re- spectively $0.5 \mathrm{ML}$ and $0.75 \mathrm{ML}$, as shown in Fig. 2(b), (c), (e) and (f). This allows us to perform DFT calculations of the relaxed structures where all Sn atoms have just a slight preference (by 0.03/0.02 eV) for hcp hollow sites compared to fcc ones-, and to simulate their corre- sponding STM images (Fig. 2(d) and (g)). As clearly seen, they show zigzag chains with two $S n$ atoms, labelled 1 and 2 per $(2 \times \sqrt{3})$ cell, having the same environment, and a honeycomb-like structure with three $\mathrm{Sn}$ atoms labelled 1, 2 and 3 per cell, where atom 2 has a different environment, in excellent agreement with the STM observations. As both zigzag chain-like and honeycomb-like arrangements are observed, indicating local Sn adatom density fluctuations at the 0.6 ML coverage, clearly both structures are energetically stable, which is confirmed in our DFT calculations where the difference is less than $0.05 \mathrm{eV}$.
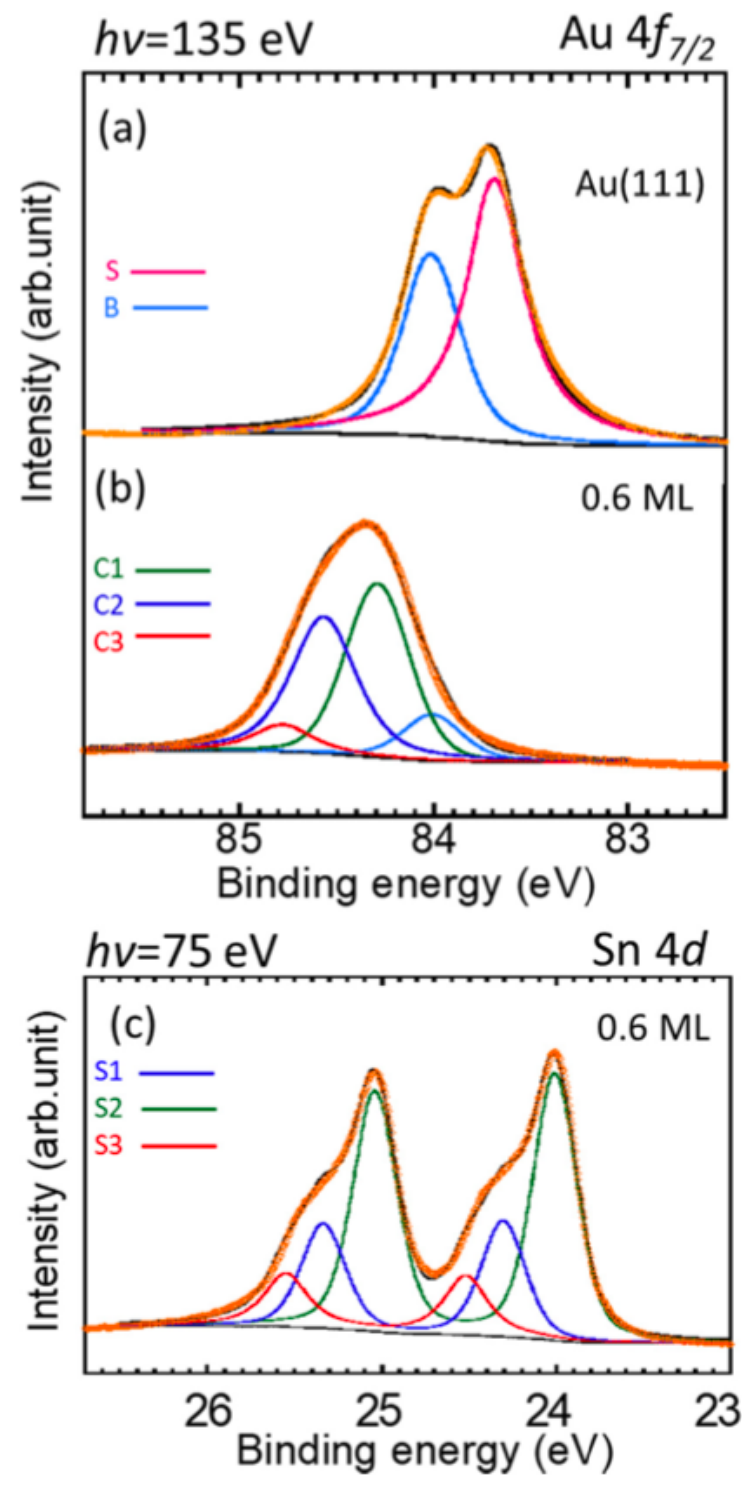

Fig. 3. The core level spectra of $A u 4 f$ and $S n 4 d$ measured at $10 \mathrm{~K}$ and taken at hv $=135 \mathrm{eV}$ and $75 \mathrm{eV}$, respectively. (a) Au 4f7/2 for a clean bare $A u(111)$ single crystal, (b) and (c) $A u$ $4 \mathrm{f} 7 / 2$ and $\mathrm{Sn} 4 \mathrm{~d}$ of stanene on $\mathrm{Au}(111)$ surface. The orange circle line is the fit line, and the black line is the experiment's data. 
As shown in Fig. 3, we further performed high-resolution synchro- tron radiation core-level (CL) spectroscopy of the shallow Sn $4 \mathrm{~d}$ and Au $4 \mathrm{f} C L$ 's in highly surface sensitive conditions. A spectrum recorded from the clean $\mathrm{Au}(111)$ surface fitted with two components (bulk: $B$ at 84.02 $\mathrm{eV}$ binding energy (BE) and surface: $\mathrm{S}$ at $83.69 \mathrm{eV} \mathrm{BE}$ ones), in excellent agreement with the literature [36]) is shown in Fig. 3(a). After the deposition of $0.6 \mathrm{ML}$ Sn giving the " $2 \times \sqrt{3}$ " superstructure, the corresponding Au 4f7/2 photoemission spectrum is presented in Fig. 3(b). The $S$ surface component has vanished, but new components, located at the higher binding energy side of the bulk component, have appeared. There are three components labelled $\mathrm{C} 1$, $\mathrm{C} 2$ and $\mathrm{C} 3$ at $84.29,84.57$ and $84.78 \mathrm{eV}$ BEs, respectively. The bulk component B has markedly decreased, which is the expected behavior for a substrate core-level peak. Component C3 may be related to Au atoms that diffuse through the Sn layer forming the AuSn alloy [26]. We relate compo- nents $\mathrm{C} 1$ and $\mathrm{C} 2$ to the interface top $\mathrm{Au}(111)$ layer beneath $\mathrm{Sn}$ atoms, either in the chain-like or the honeycomb-like local arrangements.

As for the corresponding Sn 4d broad spectrum, displayed in Fig. 3(c), we fit it with three distinct components, S1, S2, and S3, re- spectively at 24.31 (Sn 4d5/2), 24.01 and $24.50 \mathrm{eV}$ BEs. The S3 com- ponent is of comparable intensity and appears at the same binding energy as for the Au-Sn alloy investigated in ref. 26, hence, it is in correspondence with C3 discussed above. The main component S2 is about twice the intensity of S1, indicating high and low Sn coverages, respectively. Therefore, we relate components $\mathrm{S} 1$ and $\mathrm{S} 2$ to the chain- like and the honeycomb-like local arrangements, respectively. This is in correspondence to the previous $\mathrm{C} 1$ and $\mathrm{C} 2 \mathrm{Au} 4 \mathrm{f} 7 / 2$ components in the interface region.

We have further investigated the electronic band structure of the " $2 \times \sqrt{3}$ " phase by ARPES measurements at a photon energy of $70 \mathrm{eV}$. The corresponding data are shown in Fig. 4. A scheme of the $\mathrm{Au}(111)$ and " $2 \times \sqrt{3}$ " surface Brillouin zones is shown in Fig. 4(a). One notices that because of the expansion and compression mentioned above, the " $2 \times \sqrt{3}$ " surface Brillouin zone is nearly a square, that is the $\Gamma^{-} \bar{X}$ Sn and $\Gamma^{-} \bar{Y}$ Sn distances are practically the same. The clean $\mathrm{Au}(111)$ surface exhibits the well-known Rashba spin splitting of the Shockley surface- state (S) around the $\Gamma^{-}$point, shown in Fig. 4 (b). With $0.6 \mathrm{Sn} \mathrm{ML}(\mathrm{S})$ has vanished, and a new parabolic surface electronic band is observed in Fig. 4 (c) and (d). We note that no signature of a Au-Sn alloy, i.e., typical $\Lambda$-shape bands around the $\Gamma$ point, is present [25]. The effective mass $\mathrm{m}^{*}$ is calculated to be $0.41 \mathrm{me}$, where me is the electron rest mass, which is significantly higher than for that of the bare $\mathrm{Au}(111)$ Shockley surface state $(0.28 \mathrm{me})$. The Fermi velocity is estimated to be $1.4 \times 10^{6} \mathrm{~m} / \mathrm{s}$. 
(a)
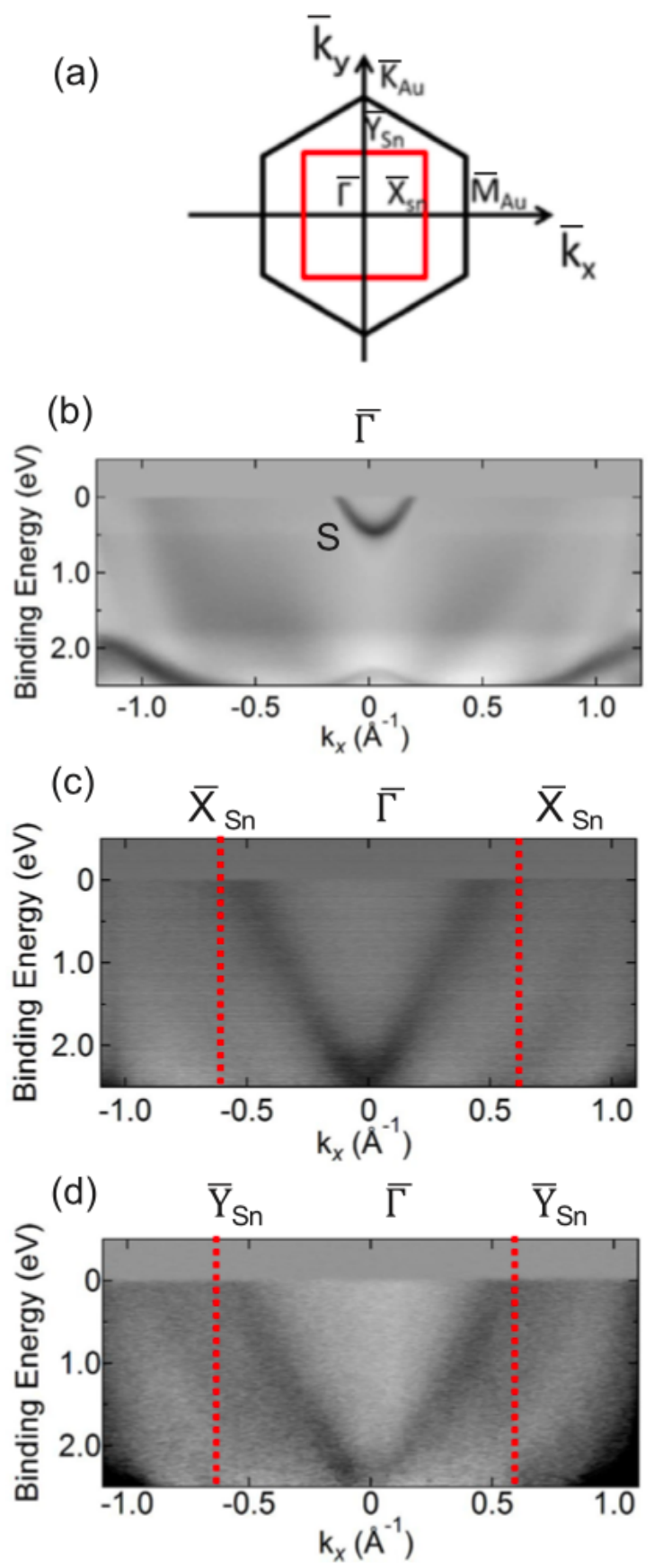

Fig. 4. ARPES dispersion at $h v=70 \mathrm{eV}$, measured at $10 \mathrm{~K}$. (a) Scheme of the Au (111) surface Brillouin zone in black and of the " $2 \times \sqrt{3}$ " Sn phase in red. (b) Shockley surface state of the clean $\mathrm{Au}(111)$ surface along the $M A u-\Gamma-M$ Au di- rection. (c) and (d) electronic band structures of the " $2 \times \sqrt{3}$ " stanene-like phase on the $A u(111)$, measured along the $M$ Au-X Sn$\Gamma^{-}-X$ Sn-M Au and $K$ Au-Y Sn- $\Gamma^{-}-Y$ Sn-K Au directions.

We have compared these experimental results to DFT calculations for the two commensurate $(2 \times \sqrt{3})$ approximant phases in Fig. 5 . The surface band structure of the clean unreconstructed $\mathrm{Au}(111) 1 \times 1$ 
surface is displayed in Fig. 5(a) in the range corresponding to $\bar{X} \mathrm{Sn}-\Gamma^{-}-\bar{X} \mathrm{Sn}$, revealing the (S) state, but significantly upward shifted. Fig. 5(b) and (c) show the calculated dispersions along $\bar{X} \mathrm{Sn}-\Gamma^{-}-\bar{X} \mathrm{Sn}$ for the $\mathrm{Sn}$ related bands of the $(2 \times \sqrt{3})$ phases at 0.5 and $0.75 \mathrm{Sn} \mathrm{ML}$, respectively. However, there is no clear correspondence with the experimental dis- persion in Fig. 4(c), except possibly for the calculation shown in Fig. 5(c) for the $0.75 \mathrm{ML}$ coverage, i.e., for the honeycomb-like struc- ture, or, in other words, the stanene-like structure, if we consider a likely up-shift. Hence, the stanene-like structure is more favorable in our experimental conditions, although, indeed, the DFT calculations for the commensurate $(2 \times \sqrt{3})$ approximant phase are just indicative.
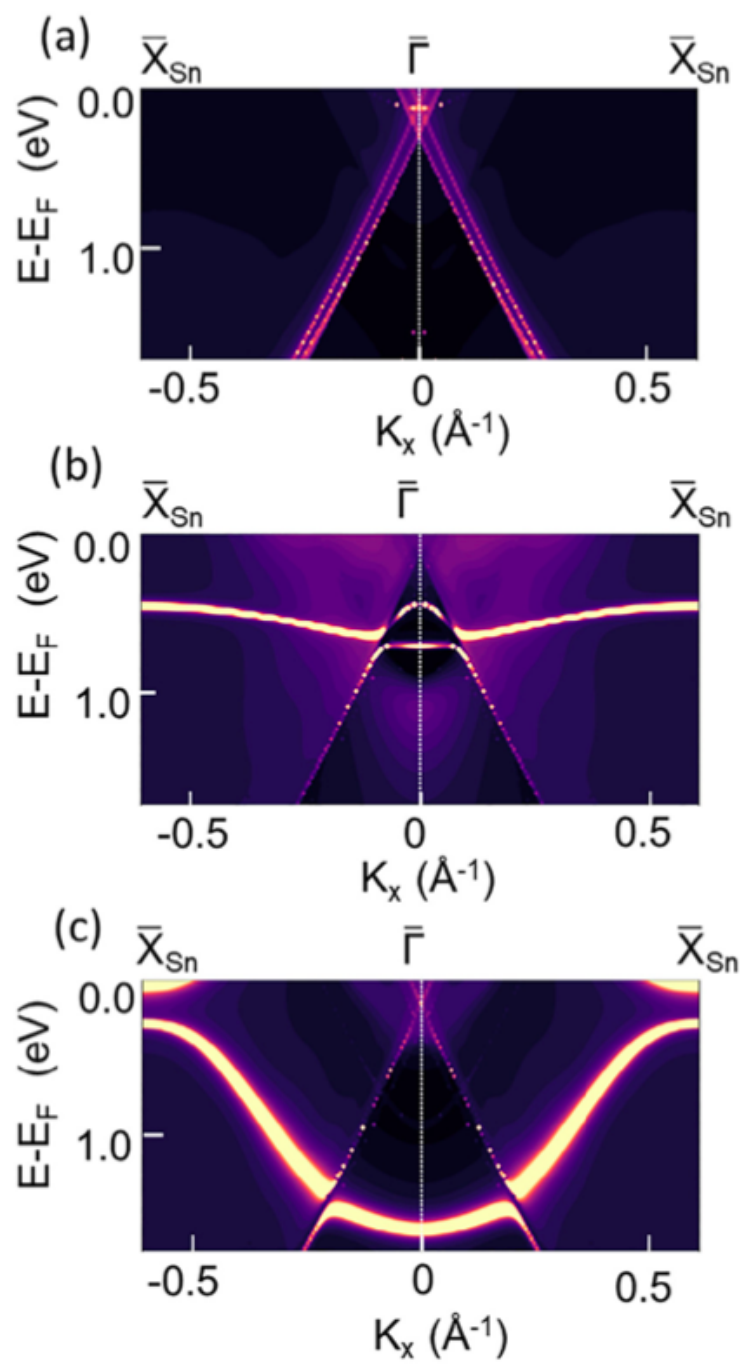

Fig. 5. (a) The calculated surface band structures along the $X S n-\Gamma-X$ Sn interval for bare $\mathrm{Au}(111) 1 \times 1$ surface. (b) The Sn-related band structure for the chain- like phase at $0.5 \mathrm{ML} \mathrm{Sn}$ coverage. (c) The Sn-related band structure for the stanene-like phase at $0.75 \mathrm{ML} S \mathrm{Sn}$ coverage.

Both experimental and theoretical parabolic dispersions clearly differ from the cone-like one expected for free-standing stanene, which is no surprise since, for one, the stanene-like Sn overlayer is not purely honeycomb, and, for two, is interacting with the $\mathrm{Au}(111)$ substrate. 
However, we stress that the structural flatness of the stanene-like phase is likely to drive a large QSH effect, possibly even at RT [16].

\section{Conclusion}

In conclusion, the tin sheet forms a nearly planar structure on the $\mathrm{Au}(111)$ surface with a " $2 \times \sqrt{3}$ " superstructure in large surface areas, identified by LEED patterns and STM images. In addition, based on experimental results, we proposed zigzag chain and honeycomb-like structures characterized by STM observations and DFT calculations. Meanwhile, the core-level spectra of $\mathrm{Sn}$ deposited onto the $\mathrm{Au}(111)$ surface reveal that a structurally flat stanene-like sheet lays almost di- rectly on $\mathrm{Au}(111)$ surface. Both experimental and theoretical band structures favor a stanene-like honeycomb structure with three $\mathrm{Sn}$ atoms per unit cell.

\section{Acknowledgments}

The authors are grateful to Nagoya University Synchrotron Radiation Research Center for financial support for ARPES measure- ments, which were conducted at BL7U of Aichi Synchrotron Radiation Center, Aichi Science \& Technology Foundation. JY acknowledges financial support from Murata Science Foundation in 2019. GLL ac- knowledges support from Nagoya University thanks to an "Eminent Foreign Scientist" Invitation Award in 2015-2016 and 2019-2020, as well as an Invitational Fellowship for Research in Japan by the Japan Society for the Promotion of Science (JSPS) in 2017. M.A. is partly supported by JSPS KAKENHI Grant Number $19 \mathrm{H} 04541$. The computa- tion in this work has been done in part using the facilities at the Information Technology Center, Nagoya University.

\section{References}

[1] D. Pesin, A.H. MacDonald, Spintronics and pseudospintronics in graphene and to- pological insulators, Nat. Mater. 11 (2012) 409-416, https://doi.org/10.1038/ nmat3305.

[2] C.L. Kane, E.J. Mele, Quantum spin hall effect in graphene, Phys. Rev. Lett. 95 (2005) 226801, https://doi.org/10.1103/PhysRevLett.95.226801.

[3] Y. Xu, B. Yan, H.-J. Zhang, J. Wang, G. Xu, P. Tang, W. Duan, S.-C. Zhang, Large-gap quantum spin hall insulators in tin films, Phys. Rev. Lett. 111 (2013) 136804, https://doi.org/10.1103/PhysRevLett.111.136804.

[4] Y. Ouyang, J. Guo, A theoretical study on thermoelectric properties of graphene nanoribbons, Appl. Phys. Lett. 94 (2009) 263107, https://doi.org/10.1063/1. 3171933.

[5] J. Yuhara, B. He, N. Matsunami, M. Nakatake, G. Le Lay, Graphene's latest cousin: plumbene epitaxial growth on a "Nano WaterCube", Adv. Mater. 31 (2019) 1901017, https://doi.org/10.1002/adma.201901017.

[6] M. Ezawa, Monolayer topological insulators: silicene, germanene, and stanene, J. Phys. Soc. Jpn. 84 (2015) 121003, https://doi.org/10.7566/JPSJ.84.121003.

[7] Z. Ni, E. Minamitani, Y. Ando, S. Watanabe, Germanene and stanene on two-dimensional substrates: Dirac cone and Z 2 invariant, Phys. Rev. B. 96 (2017) 075427, https://doi.org/10.1103/PhysRevB.96.075427. 
[8] S. Rachel, M. Ezawa, Giant magnetoresistance and perfect spin filter in silicene, germanene, and stanene, Phys. Rev. B. 89 (2014) 195303, https://doi.org/10.1103/PhysRevB.89.195303.

[9] J.-C. Rojas-Sánchez, S. Oyarzún, Y. Fu, A. Marty, C. Vergnaud, S. Gambarelli, L. Vila, M. Jamet, Y. Ohtsubo, A. Taleb-lbrahimi, P. Le Fèvre, F. Bertran, N. Reyren, J.-M. George, A. Fert, Spin to charge conversion at room temperature by spin pumping into a new type of topological insulator: a -Sn Films, Phys. Rev. Lett. 116 (2016) 096602, https://doi.org/10.1103/PhysRevLett.116.096602.

[10] B. Mortazavi, O. Rahaman, M. Makaremi, A. Dianat, G. Cuniberti, T. Rabczuk, Firstprinciples investigation of mechanical properties of silicene, germanene and sta- nene, Physica E 87 (2017) 228-232, https://doi.org/10.1016/j.physe.2016.10.047.

[11] X. Zheng, J.-F. Zhang, B. Tong, R.-R. Du, Epitaxial growth and electronic properties of few-layer stanene on InSb (111), 011001, 2D Mater 7 (2019), https://doi.org/10. 1088/20531583/ab42b9.

[12] C.-Z. Xu, Y.-H. Chan, P. Chen, X. Wang, D. Flötotto, J.A. Hlevyack, G. Bian, S.-K. Mo, M.-Y. Chou, T.-C. Chiang, Gapped electronic structure of epitaxial stanene on $\operatorname{lnSb}(111)$, Phys. Rev. B. 97 (2018) 035122, https://doi.org/10.1103/PhysRevB.97. 035122.

[13] F. Zhu, W. Chen, Y. Xu, C. Gao, D. Guan, C. Liu, D. Qian, S.-C. Zhang, J. Jia, Epitaxial growth of two-dimensional stanene, Nature Mater. 14 (2015) 1020-1025, https://doi.org/10.1038/nmat4384.

[14] P. Li, Stanene on a SiC(0001) surface: a candidate for realizing quantum anomalous Hall effect, Phys. Chem. Chem. Phys. 21 (2019) 11150-11157, https://doi.org/10. 1039/C9CP01509D.

[15] K.-C. Chen, L.-M. Lee, H.-A. Chen, H. Sun, C.-L. Wu, H.-A. Chen, K.-B. Lin, Y.-C. Tseng, C.-C. Kaun, C.-W. Pao, S.-Y. Lin, Multi-layer elemental 2D materials: antimonene, germanene and stanene grown directly on molybdenum disulfides, Semicond. Sci. Technol. 34 (2019) 105020, https://doi.org/10.1088/1361-6641/ ab3c8a.

[16] J. Gou, L. Kong, H. Li, Q. Zhong, W. Li, P. Cheng, L. Chen, K. Wu, Strain-induced band engineering in monolayer stanene on Sb(111), Phys. Rev. Materials. 1 (2017) 054004, https://doi.org/10.1103/PhysRevMaterials.1.054004.

[17] J. Yuhara, Y. Fujii, K. Nishino, N. Isobe, M. Nakatake, L. Xian, A. Rubio, G. Le Lay, Large area planar stanene epitaxially grown on $\mathrm{Ag}(111), 2 \mathrm{D}$ Mater. 5 (2018) 025002. https://doi.org/10.1088/2053-1583/aa9ea0.

[18] R. Ahmed, T. Nakagawa, S. Mizuno, Structure determination of ultra-flat stanene on $\mathrm{Cu}(111)$ using low energy electron diffraction, Surf. Sci. 691 (2020) 121498, https://doi.org/10.1016/j.susc.2019.121498.

[19] P. Vishnoi, K. Pramoda, C.N.R. Rao, 2D Elemental Nanomaterials Beyond Graphene, ChemNanoMat 5 (2019) 1062-1091, https://doi.org/10.1002/cnma.201900176. 
[20] J. Lyu, S. Zhang, C. Zhang, P. Wang, Stanene: A Promising Material for New Electronic and Spintronic Applications, Ann. Phys. 531 (2019) 1900017, https:// doi.org/10.1002/andp.201900017.

[21] S. Nigam, S. Gupta, D. Banyai, R. Pandey, C. Majumder, Evidence of a graphene-like Snsheet on a $\mathrm{Au}(111)$ substrate: electronic structure and transport properties from first principles calculations, Phys. Chem. Chem. Phys. 17 (2015) 6705-6712, https://doi.org/10.1039/C4CP04861J.

[22] M.-G. Barthès, C. Pariset, A low energy electron diffraction-Auger electron spec- troscopy study of alloy formation during the adsorption of tin on (100) and (111) Au, Thin Solid Films 77 (1981) 305-312, https://doi.org/10.1016/0040-6090(81) 90322-9.

[23] M. Maniraj, B. Stadtmüller, D. Jungkenn, M. Düvel, S. Emmerich, W. Shi, J. Stöckl, L. Lyu, J. Kollamana, Z. Wei, A. Jurenkow, S. Jakobs, B. Yan, S. Steil, M. Cinchetti, S. Mathias, M. Aeschlimann, A case study for the formation of stanene on a metal surface, Commun Phys. 2 (2019) 12, https://doi.org/10.1038/s42005-019-0111-2.

[24] Y. Liu, N. Gao, J. Zhuang, C. Liu, J. Wang, W. Hao, S.X. Dou, J. Zhao, Y. Du, Realization of strained stanene by interface engineering, J. Phys. Chem. Lett. 10 (2019) 1558-1565, https://doi.org/10.1021/acs.jpclett.9b00348.

[25] P. Sadhukhan, D. Pandey, V.K. Singh, S. Sarkar, A. Rai, K. Bhattacharya,

A. Chakrabarti, S. Roy Barman, Electronic structure and morphology of thin surface alloy layers formed by deposition of Sn on Au(111), Appl. Surf. Sci. 506 (2020) 144606, https://doi.org/10.1016/j.apsusc.2019.144606.

[26] P. Sadhukhan, S. Barman, T. Roy, V.K. Singh, S. Sarkar, A. Chakrabarti,

S.R. Barman, Electronic structure of Au-Sn compounds grown on Au(111), Phys. Rev. B. 100 (2019) 235404, https://doi.org/10.1103/PhysRevB.100.235404.

[27] D. Zhou, N. Si, B. Jiang, X. Song, H. Huang, Q. Ji, T. Niu, Interfacial effects on the growth of atomically thin film: Group VA elements on Au(111), Adv. Mater. Interfaces. 6 (2019) 1901050, https://doi.org/10.1002/admi.201901050.

[28] J. Yuhara, M. Inoue, K. Morita, Phase transition of the $\mathrm{Si}(111)-A u$ surface from $\sqrt{3} \times \sqrt{3}$ to $5 \times 1$ structure studied by means of the low-energy electron diffraction, Auger electron spectroscopy, and Rutherford backscattering spectroscopy techniques, J. Vac. Sci. Technol., A 10 (1992) 334-338, https://doi.org/10.1116/1.578053.

[29] J. Yuhara, K. Morita, J. Falta, B.H. Müller, M. Horn-von Hoegen, Characterization ofGe $\delta-$ doped Si(111) with RBS channeling, Surface and Interface, Analysis. 31 (2001)754-760, https://doi.org/10.1002/sia.1106.

[30] https://www.synopsys.com/silicon/quantumatk.html.

[31] M.J. van Setten, M. Giantomassi, E. Bousquet, M.J. Verstraete, D.R. Hamann, X. Gonze, G.-M. Rignanese, The PseudoDojo: Training and grading a 85 element optimized normconserving pseudopotential table, Comput. Phys. Commun. 226 (2018) 39-54, https://doi.org/10.1016/j.cpc.2018.01.012. 
[32] http://www.pseudo-dojo.org/.

[33] J.P. Perdew, K. Burke, M. Ernzerhof, Generalized Gradient Approximation Made Simple, Phys. Rev. Lett. 77 (1996) 3865-3868, https://doi.org/10.1103/ PhysRevLett.77.3865.

[34] M. Brandbyge, J.-L. Mozos, P. Ordejón, J. Taylor, K. Stokbro, Density-functional method for nonequilibrium electron transport, Phys. Rev. B. 65 (2002) 165401, https://doi.org/10.1103/PhysRevB.65.165401.

[35] J.V. Barth, H. Brune, G. Ertl, R.J. Behm, Scanning tunneling microscopy observa-tions on the reconstructed $\mathrm{Au}(111)$ surface: Atomic structure, long-range super- structure, rotational domains, and surface defects, Phys. Rev. B. 42 (1990) 9307-9318, https://doi.org/10.1103/PhysRevB.42.9307.

[36] K. Heister, M. Zharnikov, M. Grunze, L.S.O. Johansson, Adsorption of Alkanethiols and Biphenylthiols on Au and Ag Substrates: A High-Resolution X-ray photoelectron spectroscopy study, J. Phys. Chem. B. 105 (2001) 4058-4061, https://doi.org/10. 1021/jp010127q. 